https://doi.org/10.15407/ujpe64.6.477

V.I. VASKIVSKYI (В.I. ВАСЬКІВСЬКИЙ)

G. Nadjakov Institute of Solid State Physics, Bulgarian Academy of Sciences

(72, Tzarigradsko Highway, Sofia 1784,Bulgaria; e-mail: socyst@yandex.ru)

\title{
THIRD-ORDER CORRELATION FUNCTIONS FOR A COULOMB PAIR
}

\begin{abstract}
Third-order correlation functions for two particles with the electrostatic interaction have been obtained for the first time using the direct algebraic method. The main relations for the correlation functions that do not depend on the explicit form of the interaction potential between particles, as well as the relations that appear for four specific forms of the interaction operator, are considered.

Keywords: secondary quantization, Coulomb pairing, correlation functions.
\end{abstract}

\section{Introduction}

In this paper, the consideration of the exact theory of a Coulomb pair, the fundamentals of which were published in work [1], is continued. Recall that this theory is based on a modification of the direct algebraic method (DAM), which was proposed in work [2] for the determination of correlation functions. The correlation between two particles that form a Coulomb pair is a purely quantum-mechanical effect, which cannot be satisfactorily described with the help of the perturbation theory methods.

Among other methods, the method of Green's functions has to be mentioned, which was widely used before the appearance of the DAM. In essence, the DAM was developed in order to overcome the shortcomings of the Green's function method [2]. The main shortcoming consists in that the equations for Green's functions of lower orders include unknown Green's functions of higher orders. Attempts to avoid this difficulty are reduced to replacing unknown Green's functions of higher orders by approximate expressions. As a result, the potentially precise method is transformed into an approximate one.

It is worth to mention an alternative semiphenomenological method on the basis of the Landau concept of a Fermi liquid, which was developed, in particular, by the Ukrainian school [3-6]. It can also describe the effects of particle pairing and correlation. The Bogolyubov transformations [7] and various models such as the Hubbard model [8] and others (see, e.g., [9]) are also widely used. However, the task

(c) V.I. VASKIVSKYI (В.I. ВАСЬКІВСЬКИЙ), 2019

ISSN 2071-0194. Ukr. J. Phys. 2019. Vol. 64, No. 6 of composing a detailed bibliography concerning the application of various methods and models for describing the correlation effects goes beyond the scope of this article.

The remarks above were made only to emphasize the challenging character that the development of the direct algebraic method has with respect to the problem of finding the correlation functions. This method is a subject of the presented consideration. The results that have already been obtained in the secondorder approximation [1] make it possible to continue further the development of the exact theory describing the Coulomb pair.

In this work, the attention is focused on finding the correlation functions of the third order and the corresponding relations that arise in the third-order approximation. Recall that, in the case of a system consisting of two different particles with the electrostatic interaction between them, the DAM method is based on the expansion of the particle operators in the two-operator basis. In addition, the fact is used that the product of two operators is not changed at their permutation. This invariance makes it possible to generate various relations for correlation functions.

The structure of the work is as follows. First, the equations of motion of the third order for the creation and annihilation operators are considered. Then the basic relations for third-order correlation functions that do not depend on the explicit form of the particle interaction potential are analyzed. Finally, four possible forms of the interaction operator and the corresponding relations for the correlation functions that arise at that are considered. This paper 
contains results obtained for the first time using the DAM method in the third order.

\section{Equations for Operators}

Recall once more that, in this work, a modification of the direct algebraic method for finding the correlation functions, which was proposed in work [2], is considered. For this purpose, the equations of motion for the creation and annihilation operators are used. Then a system consisting of two different particles that interact electrostatically will be considered. The Hamiltonian of this system in the secondary quantization representation [10] has the form

$H=H_{1}+H_{2}+H_{12}$,

where

$H_{i}=\sum_{p} \varepsilon_{i p} a_{i p}^{+} a_{i p}$

is the Hamiltonian of a free particle of the $i$-th kind, and

$H_{12}=\sum_{p_{1}+p_{2}=p_{1}^{\prime}+p_{2}^{\prime}} U_{p_{1}^{\prime} p_{2}^{\prime} p_{2} p_{1}} a_{1 p_{1}^{\prime}}^{+} a_{2 p_{2}^{\prime}}^{+} a_{2 p_{2}} a_{1 p_{1}}$

is the interaction Hamiltonian. Here, $\varepsilon_{i p}$ is the kinetic energy, $a_{i p}^{+}$are the creation operators, $a_{i p}$ the annihilation operators, the subscripts $p=\left(s_{z}, \mathbf{p}\right)$ denote the spin projection $s_{z}$ and the momentum $\mathbf{p}=\left(p_{x}, p_{y}, p_{z}\right)$ of the particles, and $U_{p_{1}^{\prime} p_{2}^{\prime} p_{2} p_{1}}$ is the potential of interaction energy in the momentum representation. The both particles are assumed to be fermions, for which the following anticommutation rules are satisfied:

$a_{i p}^{+} a_{i q}+a_{i q} a_{i p}^{+}=\delta_{p q}$,

$a_{i p} a_{i q}+a_{i q} a_{i p}=0$,

$a_{i p}^{+} a_{i q}^{+}+a_{i q}^{+} a_{i p}^{+}=0$.

where $\delta_{p q}$ is the Kronecker delta. The fact that the creation and annihilation operators for particles of different kinds commute with each other will be used below.

From the Hamiltonian, we obtain the following equations of motion, which form the basis of the method:

$\left[a_{j q}, H\right]=K_{11 q}^{(j)} a_{j q}+K_{12 q}^{(j)} b_{j q}$,
$\left[b_{j q}, H\right]=K_{22 q}^{(j)} b_{j q}$ $\left[a_{j q}^{+}, H\right]=-K_{11 q}^{(j)} a_{j q}^{+}-K_{12 q}^{(j)} b_{j q}^{+}$,

$\left[b_{j q}^{+}, H\right]=-K_{22 q}^{(j)} b_{j q}^{+}$.

Here, the square brackets $[\ldots, \ldots]$ mean commutators,

$b_{j q}=\frac{1}{K_{12 q}^{(j)}} \sum_{p_{1}+p_{2}=p_{1}^{\prime}+p_{2}^{\prime}} U_{p_{1}^{\prime} p_{2}^{\prime} p_{2} p_{1}}\left(\delta_{2 j} \delta_{p_{2}^{\prime} q} a_{1 p_{1}^{\prime}}^{+}+\right.$

$\left.+\delta_{1 j} \delta_{p_{1}^{\prime} q} a_{2 p_{2}^{\prime}}^{+}\right) a_{2 p_{2}} a_{1 p_{1}}$

$b_{j q}^{+}=\frac{1}{K_{12 q}^{(j)}} \sum_{p_{1}+p_{2}=p_{1}^{\prime}+p_{2}^{\prime}} U_{p_{1}^{\prime} p_{2}^{\prime} p_{2} p_{1}}^{+} a_{1 p_{1}^{\prime}}^{+} a_{2 p_{2}^{\prime}}^{+} \times$

$\times\left(a_{2 p_{2}} \delta_{1 j} \delta_{p_{1} q}+a_{1 p_{1}} \delta_{2 j} \delta_{p_{2} q}\right)$,

$K_{11 q}^{(j)}=\varepsilon_{j q}, K_{12 q}^{(j)}=1$, and $K_{22 q}^{(j)}=K_{11 q}^{(j)} \pm K$, where $K \neq 0$ is an unknown constant, which is to be found [1]. The key point of the direct algebraic method is the expansion of operators in the two-operator basis, which is analogous to the expansion of vectors. In our case, the equations for the annihilation operators are expanded in the $\left(a_{i p}, b_{j q}\right)$ basis, and the Hermitian conjugate equations for the creation operators are expanded in the $\left(a_{i p}^{+}, b_{j q}^{+}\right)$basis. Below, it is assumed that $j \neq i$ in all equations and relations.

Using the operator identity

$[A B, H]=[A, H] B+A[B, H]$

the following useful equations of motion of the third order for the anticommutators $[\ldots, \ldots]_{+}$of two operators are obtained:

$\left[\left[a_{i p}^{+}, a_{i q}\right]_{+} a_{j}, H\right]=\left(\varepsilon_{i q}-\varepsilon_{i p}+\varepsilon_{j}\right)\left[a_{i p}^{+}, a_{i q}\right]_{+} a_{j}+$
$+\left[a_{i p}^{+}, b_{i q}\right]_{+} a_{j}+\left[a_{i p}^{+}, a_{i q}\right]_{+} b_{j}-\left[b_{i p}^{+}, a_{i q}\right]_{+} a_{j}$,
$\left[\left[b_{i p}^{+}, a_{i q}\right]_{+} a_{j}, H\right]=\left(\varepsilon_{i q}-K_{22 p}^{(i)}+\varepsilon_{j}\right)\left[b_{i p}^{+}, a_{i q}\right]_{+} a_{j}+$
$+\left[b_{i p}^{+}, b_{i q}\right]_{+} a_{j}+\left[b_{i p}^{+}, a_{i q}\right]_{+} b_{j}$,

$\left[\left[a_{i p}^{+}, b_{i q}\right]_{+} a_{j}, H\right]=\left(K_{22 q}^{(i)}-\varepsilon_{i p}+\varepsilon_{j}\right)\left[a_{i p}^{+}, b_{i q}\right]_{+} a_{j}+$

$+\left[a_{i p}^{+}, b_{i q}\right]_{+} b_{j}-\left[b_{i p}^{+}, b_{i q}\right]_{+} a_{j}$

$\left[\left[a_{i p}^{+}, a_{i q}\right]_{+} b_{j}, H\right]=\left(K_{22}^{(j)}-\varepsilon_{i p}+\varepsilon_{i q}\right)\left[a_{i p}^{+}, a_{i q}\right]_{+} b_{j}+$

$+\left[a_{i p}^{+}, b_{i q}\right]_{+} b_{j}-\left[b_{i p}^{+}, a_{i q}\right]_{+} b_{j}$

$\left[\left[a_{i p}^{+}, a_{i q}\right]_{+} a_{j}^{+}, H\right]=\left(\varepsilon_{i q}-\varepsilon_{i p}-\varepsilon_{j}\right)\left[a_{i p}^{+}, a_{i q}\right]_{+} a_{j}^{+}+$

$+\left[a_{i p}^{+}, b_{i q}\right]_{+} a_{j}^{+}-\left[a_{i p}^{+}, a_{i q}\right]_{+} b_{j}^{+}-\left[b_{i p}^{+}, a_{i q}\right]_{+} a_{j}^{+}$, 


$$
\begin{aligned}
& {\left[\left[b_{i p}^{+}, a_{i q}\right]_{+} a_{j}^{+}, H\right]=\left(\varepsilon_{i q}-K_{22 p}^{(i)}-\varepsilon_{j}\right)\left[b_{i p}^{+}, a_{i q}\right]_{+} a_{j}^{+}+} \\
& +\left[b_{i p}^{+}, b_{i q}\right]_{+} a_{j}^{+}-\left[b_{i p}^{+}, a_{i q}\right]_{+} b_{j}^{+}, \\
& {\left[\left[a_{i p}^{+}, b_{i q}\right]_{+} a_{j}^{+}, H\right]=\left(K_{22 q}^{(i)}-\varepsilon_{i p}-\varepsilon_{j}\right)\left[a_{i p}^{+}, b_{i q}\right]_{+} a_{j}^{+}-} \\
& -\left[a_{i p}^{+}, b_{i q}\right]_{+} b_{j}^{+}-\left[b_{i p}^{+}, b_{i q}\right]_{+} a_{j}^{+}, \\
& {\left[\left[a_{i p}^{+}, a_{i q}\right]_{+} b_{j}^{+}, H\right]=\left(\varepsilon_{i q}-\varepsilon_{i p}-K_{22}^{(j)}\right)\left[a_{i p}^{+}, a_{i q}\right]_{+} b_{j}^{+}+} \\
& +\left[a_{i p}^{+}, b_{i q}\right]_{+} b_{j}^{+}-\left[b_{i p}^{+}, a_{i q}\right]_{+} b_{j}^{+}, \\
& {\left[a_{j}\left[a_{i p}^{+}, a_{i q}\right]_{+}, H\right]=\left(\varepsilon_{i q}-\varepsilon_{i p}+\varepsilon_{j}\right) a_{j}\left[a_{i p}^{+}, a_{i q}\right]_{+}+} \\
& +a_{j}\left[a_{i p}^{+}, b_{i q}\right]_{+}+b_{j}\left[a_{i p}^{+}, a_{i q}\right]_{+}-a_{j}\left[b_{i p}^{+}, a_{i q}\right]_{+}, \\
& {\left[a_{j}\left[b_{i p}^{+}, a_{i q}\right]_{+}, H\right]=\left(\varepsilon_{i q}-K_{22 p}^{(i)}+\varepsilon_{j}\right) a_{j}\left[b_{i p}^{+}, a_{i q}\right]_{+}+} \\
& +a_{j}\left[b_{i p}^{+}, b_{i q}\right]_{+}+b_{j}\left[b_{i p}^{+}, a_{i q}\right]_{+}, \\
& {\left[a_{j}\left[a_{i p}^{+}, b_{i q}\right]_{+}, H\right]=\left(K_{22 q}^{(i)}-\varepsilon_{i p}+\varepsilon_{j}\right) a_{j}\left[a_{i p}^{+}, b_{i q}\right]_{+}+} \\
& +b_{j}\left[a_{i p}^{+}, b_{i q}\right]_{+}-a_{j}\left[b_{i p}^{+}, b_{i q}\right]_{+}, \\
& {\left[b_{j}\left[a_{i p}^{+}, a_{i q}\right]_{+}, H\right]=\left(K_{22}^{(j)}-\varepsilon_{i p}+\varepsilon_{i q}\right) b_{j}\left[a_{i p}^{+}, a_{i q}\right]_{+}+} \\
& +b_{j}\left[a_{i p}^{+}, b_{i q}\right]_{+}-b_{j}\left[b_{i p}^{+}, a_{i q}\right]_{+} \text {, } \\
& {\left[a_{j}^{+}\left[a_{i p}^{+}, a_{i q}\right]_{+}, H\right]=\left(\varepsilon_{i q}-\varepsilon_{i p}-\varepsilon_{j}\right) a_{j}^{+}\left[a_{i p}^{+}, a_{i q}\right]_{+}+} \\
& +a_{j}^{+}\left[a_{i p}^{+}, b_{i q}\right]_{+}-b_{j}^{+}\left[a_{i p}^{+}, a_{i q}\right]_{+}-a_{j}^{+}\left[b_{i p}^{+}, a_{i q}\right]_{+}, \\
& {\left[a_{j}^{+}\left[b_{i p}^{+}, a_{i q}\right]_{+}, H\right]=\left(\varepsilon_{i q}-K_{22 p}^{(i)}-\varepsilon_{j}\right) a_{j}^{+}\left[b_{i p}^{+}, a_{i q}\right]_{+}+} \\
& +a_{j}^{+}\left[b_{i p}^{+}, b_{i q}\right]_{+}-b_{j}^{+}\left[b_{i p}^{+}, a_{i q}\right]_{+}, \\
& {\left[a_{j}^{+}\left[a_{i p}^{+}, b_{i q}\right]_{+}, H\right]=\left(K_{22 q}^{(i)}-\varepsilon_{i p}-\varepsilon_{j}\right) a_{j}^{+}\left[a_{i p}^{+}, b_{i q}\right]_{+}-} \\
& -b_{j}^{+}\left[a_{i p}^{+}, b_{i q}\right]_{+}-a_{j}^{+}\left[b_{i p}^{+}, b_{i q}\right]_{+} \\
& {\left[b_{j}^{+}\left[a_{i p}^{+}, a_{i q}\right]_{+}, H\right]=\left(\varepsilon_{i q}-\varepsilon_{i p}-K_{22}^{(j)}\right) b_{j}^{+}\left[a_{i p}^{+}, a_{i q}\right]_{+}+} \\
& +b_{j}^{+}\left[a_{i p}^{+}, b_{i q}\right]_{+}-b_{j}^{+}\left[b_{i p}^{+}, a_{i q}\right]_{+} .
\end{aligned}
$$

This is not a complete set of equations of motion for the third-order operators, but only those of them, which will be used below. The obtained equations make it possible to derive equations for the thirdorder operators themselves. The corresponding detailed calculations are presented in Appendix.

ISSN 2071-0194. Ukr. J. Phys. 2019. Vol. 64, No. 6

\section{Basic Relations}

For any operator $A$, the following operators can be introduced:

$\breve{A}=\rho^{-1} A \rho$,

$\widehat{A}=\rho A \rho^{-1}$,

where $\rho$ is the statistical operator of the system. Then the following expansions can be used:

$\breve{a}_{i p}=a_{i p}-G_{i} b_{i p}$

$\breve{b}_{i p}=b_{i p}$

$\widehat{a}_{i p}=a_{i p}+G_{i} b_{i p}$,

$\widehat{b}_{i p}=b_{i p}$

$\vec{a}_{i p}^{+}=a_{i p}^{+}+G_{i} b_{i p}^{+}$

$\breve{b}_{i p}^{+}=b_{i p}^{+}$

$\widehat{a}_{i p}^{+}=a_{i p}^{+}-G_{i} b_{i p}^{+}$,

$\widehat{b}_{i p}^{+}=b_{i p}^{+}$,

where $G_{i}$ are unknown constants. These expansions play a substantial role while applying the method of work [1].

Let us introduce the following notation for the mean value of an arbitrary operator $A$ :

$\langle A\rangle=\operatorname{Sp}(\rho A)$,

where Sp stands for the operator trace. By applying the formula

$\operatorname{Sp}(A B)=\operatorname{Sp}(B A)$

for two arbitrary operators $A$ and $B$, the following identities for the product of three operators can be found:

$\langle A B C\rangle=\langle B C \widehat{A}\rangle=\langle\breve{C} A B\rangle$.

Since the statistical operator $\rho$ of the system is self-conjugate, Eqs. (38) and (39) immediately bring about the relations

$\left\langle a_{i p}^{+}\right\rangle=\left\langle a_{i p}\right\rangle^{+}=\overline{\left\langle a_{i p}\right\rangle}$, 
where the bar means the complex conjugation operation. Relation (40) for the correlation functions can be used to find the correlation functions themselves. In particular, from

$$
\begin{aligned}
& \left\langle a_{i p} b_{i q} b_{j}\right\rangle=\left\langle b_{i q} b_{j} a_{i p}\right\rangle+G_{i}\left\langle b_{i q} b_{j} b_{i p}\right\rangle=\left\langle b_{j} a_{i p} b_{i q}\right\rangle+ \\
& +G_{i}\left\langle b_{j} b_{i p} b_{i q}\right\rangle=\left\langle a_{i p} b_{i q} b_{j}\right\rangle+G_{i}\left\langle b_{i p} b_{i q} b_{j}\right\rangle
\end{aligned}
$$

we obtain

$\left\langle b_{i p} b_{i q} b_{j}\right\rangle=0$.

In the same way, we can verify that the mean value of the product of three operators $b$ and/or $b^{+}$taken in any combination equals zero. This is a direct consequence of the triangle form of the expansion matrices.

Then we find

$$
\begin{aligned}
& \left\langle a_{i p} a_{i q} b_{j}\right\rangle=\left\langle a_{i q} b_{j} a_{i p}\right\rangle+G_{i}\left\langle a_{i q} b_{j} b_{i p}\right\rangle= \\
& =\left\langle a_{i p} a_{i q} b_{j}\right\rangle+G_{i}\left\langle b_{i p} a_{i q} b_{j}\right\rangle+G_{i}\left\langle a_{i p} b_{i q} b_{j}\right\rangle, \\
& G_{i}\left\langle b_{i p} a_{i q} b_{j}\right\rangle+G_{i}\left\langle a_{i p} b_{i q} b_{j}\right\rangle=0 .
\end{aligned}
$$

Using the relations

$\left\langle a_{i p} b_{i q} a_{j}\right\rangle=\left\langle a_{i p} b_{i q} a_{j}\right\rangle+G_{i}\left\langle b_{i p} b_{i q} a_{j}\right\rangle+G_{j}\left\langle a_{i p} b_{i q} b_{j}\right\rangle$,

$G_{i}\left\langle b_{i p} b_{i q} a_{j}\right\rangle+G_{j}\left\langle a_{i p} b_{i q} b_{j}\right\rangle=0$,

$\left\langle b_{i p} a_{i q} a_{j}\right\rangle=\left\langle b_{i p} a_{i q} a_{j}\right\rangle+G_{i}\left\langle b_{i p} b_{i q} a_{j}\right\rangle+G_{j}\left\langle b_{i p} a_{i q} b_{j}\right\rangle$,

$G_{i}\left\langle b_{i p} b_{i q} a_{j}\right\rangle+G_{j}\left\langle b_{i p} a_{i q} b_{j}\right\rangle=0$,

we obtain

$\left\langle b_{i p} a_{i q} b_{j}\right\rangle=\left\langle a_{i p} b_{i q} b_{j}\right\rangle=\left\langle b_{i p} b_{i q} a_{j}\right\rangle=0$.

Analogously, one can verify that the mean value of the product of two operators $b$ or $b^{+}$and either of the operators $a$ or $a^{+}$, with all three being taken in an arbitrary combination, also equals zero. From the relations

$-\left\langle a_{i p} a_{i q} a_{j}\right\rangle=\left\langle a_{i q} a_{j} a_{i p}\right\rangle=\left\langle a_{i p} a_{i q} a_{j}\right\rangle-G_{i}\left\langle b_{i p} a_{i q} a_{j}\right\rangle$,

we obtain

$\left\langle b_{i p} a_{i q} a_{j}\right\rangle=2 \frac{\left\langle a_{i p} a_{i q} a_{j}\right\rangle}{G_{i}}=\left\langle a_{j} b_{i p} a_{i q}\right\rangle=\left\langle a_{i q} a_{j} b_{i p}\right\rangle$.
Analogously,

$\left\langle a_{i p} b_{i q} a_{j}\right\rangle=\left\langle b_{i q} a_{j} a_{i p}\right\rangle=\left\langle a_{j} a_{i p} b_{i q}\right\rangle=$

$=-2 \frac{\left\langle a_{j} a_{i p} a_{i q}\right\rangle}{G_{i}}=-2 \frac{\left\langle a_{i p} a_{i q} a_{j}\right\rangle}{G_{i}}$,

$\left\langle b_{i p} a_{i q} a_{j}^{+}\right\rangle=\left\langle a_{j}^{+} b_{i p} a_{i q}\right\rangle=\left\langle a_{i q} a_{j}^{+} b_{i p}\right\rangle=-\left\langle a_{i p} b_{i q} a_{j}^{+}\right\rangle=$

$=-\left\langle a_{j}^{+} a_{i p} b_{i q}\right\rangle=-\left\langle b_{i q} a_{j}^{+} a_{i p}\right\rangle=2 \frac{\left\langle a_{i p} a_{i q} a_{j}^{+}\right\rangle}{G_{i}}$.

Finally, we obtain

$\left\langle a_{i p} a_{i q} b_{j}\right\rangle=\left\langle a_{i q} b_{j} a_{i p}\right\rangle=\left\langle b_{j} a_{i p} a_{i q}\right\rangle=$

$=\left\langle a_{i p} a_{i q} b_{j}^{+}\right\rangle=\left\langle a_{i q} b_{j}^{+} a_{i p}\right\rangle=\left\langle b_{j}^{+} a_{i p} a_{i q}\right\rangle=0$.

In the same way, we arrive at the following relations:

$\left\langle b_{i q}^{+} a_{j}^{ \pm} a_{i p}^{+}\right\rangle=\left\langle a_{i p}^{+} b_{i q}^{+} a_{j}^{ \pm}\right\rangle=\left\langle a_{j}^{ \pm} a_{i p}^{+} b_{i q}^{+}\right\rangle=-\left\langle b_{i p}^{+} a_{i q}^{+} a_{j}^{ \pm}\right\rangle=$

$=-\left\langle a_{i q}^{+} a_{j}^{ \pm} b_{i p}^{+}\right\rangle=-\left\langle a_{j}^{ \pm} b_{i p}^{+} a_{i q}^{+}\right\rangle=2 \frac{\left\langle a_{i p}^{+} a_{i q}^{+} a_{j}^{ \pm}\right\rangle}{G_{i}}$,

$\left\langle a_{i p}^{+} a_{i q}^{+} b_{j}^{+}\right\rangle=\left\langle a_{i q}^{+} b_{j}^{+} a_{i p}^{+}\right\rangle=\left\langle b_{j}^{+} a_{i p}^{+} a_{i q}^{+}\right\rangle=\left\langle a_{i p}^{+} a_{i q}^{+} b_{j}\right\rangle=$

$=\left\langle a_{i q}^{+} b_{j} a_{i p}^{+}\right\rangle=\left\langle b_{j} a_{i p}^{+} a_{i q}^{+}\right\rangle=0$,

$\left\langle a_{i q} a_{j}^{ \pm} a_{i p}^{+}\right\rangle=\left\langle a_{i p}^{+} a_{i q} a_{j}^{ \pm}\right\rangle+G_{i}\left\langle b_{i p}^{+} a_{i q} a_{j}^{ \pm}\right\rangle$,

$\left\langle b_{i p}^{+} a_{i q} a_{j}^{ \pm}\right\rangle=\frac{\left\langle a_{j}^{ \pm}\right\rangle \delta_{p q}-2\left\langle a_{i p}^{+} a_{i q} a_{j}^{ \pm}\right\rangle}{G_{i}}=\left\langle a_{j}^{ \pm} b_{i p}^{+} a_{i q}\right\rangle=$

$=\left\langle a_{i q} a_{j}^{ \pm} b_{i p}^{+}\right\rangle=\left\langle a_{j}^{ \pm} a_{i q} b_{i p}^{+}\right\rangle=\left\langle a_{i q} b_{i p}^{+} a_{j}^{ \pm}\right\rangle=\left\langle b_{i p}^{+} a_{j}^{ \pm} a_{i q}\right\rangle$,

$\left\langle a_{i p}^{+} a_{j}^{ \pm} a_{i q}\right\rangle=\left\langle a_{j}^{ \pm} a_{i q} a_{i p}^{+}\right\rangle-G_{i}\left\langle b_{i q} a_{i p}^{+} a_{j}^{ \pm}\right\rangle$,

$\left\langle b_{i q} a_{i p}^{+} a_{j}^{ \pm}\right\rangle=\frac{\left\langle a_{j}^{ \pm}\right\rangle \delta_{p q}-2\left\langle a_{i p}^{+} a_{i q} a_{j}^{ \pm}\right\rangle}{G_{i}}=\left\langle a_{j}^{ \pm} b_{i q} a_{i p}^{+}\right\rangle=$

$=\left\langle a_{i p}^{+} a_{j}^{ \pm} b_{i q}\right\rangle=\left\langle a_{j}^{ \pm} a_{i p}^{+} b_{i q}\right\rangle=\left\langle a_{i p}^{+} b_{i q} a_{j}^{ \pm}\right\rangle=\left\langle b_{i q} a_{j}^{ \pm} a_{i p}^{+}\right\rangle$.

Finally, we find

$\left\langle a_{i p}^{+} a_{i q} b_{j}^{ \pm}\right\rangle=\left\langle a_{i q} b_{j}^{ \pm} a_{i p}^{+}\right\rangle=\left\langle b_{j}^{ \pm} a_{i p}^{+} a_{i q}\right\rangle=$

$=\left\langle a_{i q} a_{i p}^{+} b_{j}^{ \pm}\right\rangle=\left\langle a_{i p}^{+} b_{j}^{ \pm} a_{i q}\right\rangle=\left\langle b_{j}^{ \pm} a_{i q} a_{i p}^{+}\right\rangle=0$.

Now, by averaging Eq. (153) from Appendix, we obtain

$\left(K_{22 p}^{(i)}+K_{22 q}^{(i)}-\varepsilon_{i p}-\varepsilon_{i q}\right)\left(\left\langle a_{j}\right\rangle \delta_{p q}-2\left\langle a_{i p}^{+} a_{i q} a_{j}\right\rangle\right)=0$.

ISSN 2071-0194. Ukr. J. Phys. 2019. Vol. 64, No. 6 
Since $K_{22 p}^{(i)}+K_{22 q}^{(i)} \neq \varepsilon_{i p}+\varepsilon_{i q}$, we have

$\left\langle a_{i p}^{+} a_{i q} a_{j}\right\rangle=\left\langle a_{j}\right\rangle \frac{\delta_{p q}}{2}$.

On the same footing,

$$
\begin{aligned}
& \left\langle a_{j} a_{i p}^{+} a_{i q}\right\rangle=\left\langle a_{i q} a_{i p}^{+} a_{j}\right\rangle=\left\langle a_{j} a_{i q} a_{i p}^{+}\right\rangle= \\
& =\left\langle a_{i p}^{+} a_{j} a_{i q}\right\rangle=\left\langle a_{i q} a_{j} a_{i p}^{+}\right\rangle=\left\langle a_{j}\right\rangle \frac{\delta_{p q}}{2}, \\
& \left\langle a_{i p}^{+} a_{i q} a_{j}^{+}\right\rangle=\left\langle a_{i q} a_{i p}^{+} a_{j}^{+}\right\rangle=\left\langle a_{j}^{+} a_{i p}^{+} a_{i q}\right\rangle=\left\langle a_{j}^{+} a_{i q} a_{i p}^{+}\right\rangle= \\
& =\left\langle a_{i p}^{+} a_{j}^{+} a_{i q}\right\rangle=\left\langle a_{i q} a_{j}^{+} a_{i p}^{+}\right\rangle=\left\langle a_{j}^{+}\right\rangle \frac{\delta_{p q}}{2} .
\end{aligned}
$$

Performing analogous calculations, we can obtain expressions for the correlation functions of operators for the particles of the same kind in the case where $q \neq p$ :

$\left\langle a_{i p}^{+} a_{i q} b_{i p}\right\rangle=\left\langle b_{i p} a_{i p}^{+} a_{i q}\right\rangle=\left\langle a_{i q} b_{i p} a_{i p}^{+}\right\rangle=-\left\langle a_{i p}^{+} b_{i p} a_{i q}\right\rangle=$

$=-\left\langle b_{i p} a_{i q} a_{i p}^{+}\right\rangle=-\left\langle a_{i q} a_{i p}^{+} b_{i p}\right\rangle=\frac{\left\langle a_{i q}\right\rangle}{G_{i}}$,

$\left\langle b_{i p}^{+} a_{i q} a_{i p}\right\rangle=\left\langle a_{i p} b_{i p}^{+} a_{i q}\right\rangle=\left\langle a_{i q} a_{i p} b_{i p}^{+}\right\rangle=-\left\langle b_{i p}^{+} a_{i p} a_{i q}\right\rangle=$

$=-\left\langle a_{i q} b_{i p}^{+} a_{i p}\right\rangle=-\left\langle a_{i p} a_{i q} b_{i p}^{+}\right\rangle=\frac{\left\langle a_{i q}\right\rangle}{G_{i}}$,

$\left\langle b_{i p}^{+} a_{i q}^{+} a_{i p}\right\rangle=\left\langle a_{i p} b_{i p}^{+} a_{i q}^{+}\right\rangle=\left\langle a_{i q}^{+} a_{i p} b_{i p}^{+}\right\rangle=-\left\langle b_{i p}^{+} a_{i p} a_{i q}^{+}\right\rangle=$

$=-\left\langle a_{i q}^{+} b_{i p}^{+} a_{i p}\right\rangle=-\left\langle a_{i p} a_{i q}^{+} b_{i p}^{+}\right\rangle=\frac{\left\langle a_{i q}^{+}\right\rangle}{G_{i}}$,

$\left\langle a_{i p}^{+} a_{i q}^{+} b_{i p}\right\rangle=\left\langle a_{i q}^{+} b_{i p} a_{i p}^{+}\right\rangle=\left\langle b_{i p} a_{i p}^{+} a_{i q}^{+}\right\rangle=-\left\langle a_{i q}^{+} a_{i p}^{+} b_{i p}\right\rangle=$

$=-\left\langle a_{i p}^{+} b_{i p} a_{i q}^{+}\right\rangle=-\left\langle b_{i p} a_{i q}^{+} a_{i p}^{+}\right\rangle=\frac{\left\langle a_{i q}^{+}\right\rangle}{G_{i}}$,

$\left\langle a_{i p}^{+} a_{i p} b_{i q}\right\rangle=\left\langle a_{i p} a_{i p}^{+} b_{i q}\right\rangle=\left\langle a_{i p} b_{i q} a_{i p}^{+}\right\rangle=\left\langle a_{i p}^{+} b_{i q} a_{i p}\right\rangle=$

$=\left\langle b_{i q} a_{i p}^{+} a_{i p}\right\rangle=\left\langle b_{i q} a_{i p} a_{i p}^{+}\right\rangle=0$,

$\left\langle a_{i p}^{+} a_{i q} b_{i q}\right\rangle=\left\langle b_{i q} a_{i p}^{+} a_{i q}\right\rangle=\left\langle a_{i q} b_{i q} a_{i p}^{+}\right\rangle=\left\langle a_{i q} a_{i p}^{+} b_{i q}\right\rangle=$

$=\left\langle b_{i q} a_{i q} a_{i p}^{+}\right\rangle=\left\langle a_{i p}^{+} b_{i q} a_{i q}\right\rangle=0$,

$\left\langle a_{i p}^{+} b_{i p}^{+} a_{i q}\right\rangle=\left\langle b_{i p}^{+} a_{i q} a_{i p}^{+}\right\rangle=\left\langle a_{i q} a_{i p}^{+} b_{i p}^{+}\right\rangle=\left\langle a_{i p}^{+} a_{i q} b_{i p}^{+}\right\rangle=$

$=\left\langle b_{i p}^{+} a_{i p}^{+} a_{i q}\right\rangle=\left\langle a_{i q} b_{i p}^{+} a_{i p}^{+}\right\rangle=0$,

$\left\langle a_{i p}^{+} a_{i p} b_{i q}^{+}\right\rangle=\left\langle a_{i p} a_{i p}^{+} b_{i q}^{+}\right\rangle=\left\langle a_{i p} b_{i q}^{+} a_{i p}^{+}\right\rangle=\left\langle a_{i p}^{+} b_{i q}^{+} a_{i p}\right\rangle=$

$=\left\langle b_{i q}^{+} a_{i p}^{+} a_{i p}\right\rangle=\left\langle b_{i q}^{+} a_{i p} a_{i p}^{+}\right\rangle=0$,

$\left\langle b_{i p}^{+} a_{i q} a_{i q}\right\rangle=\left\langle a_{i q} a_{i q} b_{i p}^{+}\right\rangle=\left\langle a_{i q} b_{i p}^{+} a_{i q}\right\rangle=0$,

$\left\langle b_{i q} a_{i p}^{+} a_{i p}^{+}\right\rangle=\left\langle a_{i p}^{+} a_{i p}^{+} b_{i q}\right\rangle=\left\langle a_{i p}^{+} b_{i q} a_{i p}^{+}\right\rangle=0$.

The relations obtained from formulas (67)-(76) by replacing in them $q$ for $p$ and vice versa will also be valid.

ISSN 2071-0194. Ukr. J. Phys. 2019. Vol. 64, No. 6

\section{Relations for Central Distribution Moments}

It is useful to study the structure of correlations for central distribution moments, because the presence of a correlation for fluctuations testifies to a phase transition in the system [11]. The relations for the central distribution moments can be easily obtained by preforming a shift of the operators, namely,

$a_{i p}=\left\langle a_{i p}\right\rangle+\alpha_{i p}$

$a_{i p}^{+}=\overline{\left\langle a_{i p}\right\rangle}+\alpha_{i p}^{+}$.

The main relations for the operators $\alpha$ are as follows:

$\left\langle\left[\alpha_{i}, \alpha_{j}\right]\right\rangle=\left\langle\left[\alpha_{i}^{+}, \alpha_{j}\right]\right\rangle=\left\langle\left[\alpha_{i}^{+}, \alpha_{j}^{+}\right]\right\rangle=0$,

$\left\langle\left[\alpha_{i p}, \alpha_{i q}\right]_{+}\right\rangle=-2\left\langle a_{i p}\right\rangle\left\langle a_{i q}\right\rangle$

$\left\langle\left[\alpha_{i p}^{+}, \alpha_{i q}\right]_{+}\right\rangle=\delta_{p q}-2 \overline{\left\langle a_{i p}\right\rangle}\left\langle a_{i q}\right\rangle$,

$\left\langle\left[\alpha_{i p}^{+}, \alpha_{i q}^{+}\right]_{+}\right\rangle=-2 \overline{\left\langle a_{i p}\right\rangle\left\langle a_{i q}\right\rangle}$,

$\left\langle\left[\alpha_{j}^{ \pm}, \alpha_{i p}\right] \alpha_{i q}\right\rangle=\left\langle\left[\alpha_{j}^{ \pm}, \alpha_{i p}^{+}\right] \alpha_{i q}\right\rangle=$

$=\left\langle\alpha_{i p}\left[\alpha_{j}^{ \pm}, \alpha_{i q}\right]\right\rangle=\left\langle\alpha_{i p}^{+}\left[\alpha_{j}^{ \pm}, \alpha_{i q}\right]\right\rangle=0$,

$\left\langle\left[\alpha_{i p}, \alpha_{i q}\right]_{+} \alpha_{j}^{ \pm}\right\rangle=\left\langle\alpha_{j}^{ \pm}\left[\alpha_{i p}, \alpha_{i q}\right]_{+}\right\rangle=$

$=-2\left\langle a_{i p}\right\rangle\left\langle\alpha_{i q} \alpha_{j}^{ \pm}\right\rangle-2\left\langle a_{i q}\right\rangle\left\langle\alpha_{i p} \alpha_{j}^{ \pm}\right\rangle$,

$\left\langle\left[\alpha_{i p}^{+}, \alpha_{i q}\right]_{+} \alpha_{j}^{ \pm}\right\rangle=\left\langle\alpha_{j}^{ \pm}\left[\alpha_{i p}^{+}, \alpha_{i q}\right]_{+}\right\rangle=$

$=-2 \overline{\left\langle a_{i p}\right\rangle}\left\langle\alpha_{i q} \alpha_{j}^{ \pm}\right\rangle-2\left\langle a_{i q}\right\rangle\left\langle\alpha_{i p}^{+} \alpha_{j}^{ \pm}\right\rangle$,

$\left\langle\left[\alpha_{i p}^{+}, \alpha_{i q}^{+}\right]_{+} \alpha_{j}^{ \pm}\right\rangle=\left\langle\alpha_{j}^{ \pm}\left[\alpha_{i p}^{+}, \alpha_{i q}^{+}\right]_{+}\right\rangle=$

$=-2 \overline{\left\langle a_{i p}\right\rangle}\left\langle\alpha_{i q}^{+} \alpha_{j}^{ \pm}\right\rangle-2 \overline{\left\langle a_{i q}\right\rangle}\left\langle\alpha_{i p}^{+} \alpha_{j}^{ \pm}\right\rangle$,

$\left\langle\alpha_{i q} \alpha_{i p}^{+}\right\rangle=\left\langle\alpha_{i p}^{+} \alpha_{i q}\right\rangle=\frac{\delta_{p q}}{2}-\overline{\left\langle a_{i p}\right\rangle}\left\langle a_{i q}\right\rangle$,

$\left\langle\alpha_{i q} \alpha_{i p}^{+} \alpha_{j}^{ \pm}\right\rangle=\left\langle\alpha_{i p}^{+} \alpha_{i q} \alpha_{j}^{ \pm}\right\rangle=$

$=-\overline{\left\langle a_{i p}\right\rangle}\left\langle\alpha_{i q} \alpha_{j}^{ \pm}\right\rangle-\left\langle a_{i q}\right\rangle\left\langle\alpha_{i p}^{+} \alpha_{j}^{ \pm}\right\rangle$.

\section{Main Variants}

We have already noted [1] that every particle can possess only two different states: states with identical momenta and different spin projections or, vice versa, 
states with different momenta. Therefore, let us consider four main variants. Recall that the particles interacting electrostatically by means of the Coulomb potential

$\Phi(\mathbf{r})=\frac{Q_{1} Q_{2}}{4 \pi \varepsilon_{0} r}$

are dealt with. Here, $Q_{1}$ and $Q_{2}$ are the charges of the particles that are located at the distance $r$ from each other, and $\varepsilon_{0}$ is the absolute dielectric permittivity of the vacuum.

The first variant corresponds to the following operator wave functions of the particles:

$\Psi_{j}\left(\mathbf{r}_{j}\right)=\frac{e^{\frac{i \mathbf{p}_{j} \cdot \mathbf{r}_{j}}{\hbar}}}{\sqrt{V}}\left(a_{j+}+a_{j-}\right)$,

$\Psi_{j}^{+}\left(\mathbf{r}_{j}\right)=\frac{e^{\frac{-i \mathbf{p}_{j} \cdot \mathbf{r}_{j}}{\hbar}}}{\sqrt{V}}\left(a_{j+}^{+}+a_{j-}^{+}\right)$,

where $\mathbf{p}_{j} \cdot \mathbf{r}_{j}$ denotes the scalar product of the momentum vector $\mathbf{p}_{j}$ and the radius vector $\mathbf{r}_{j}$ of a particle of the $j$-th kind; $a_{j+}^{+}$and $a_{j+}$ are the creation and annihilation, respectively, operators for the particle with the spin projection $+\frac{1}{2} \hbar$, whereas $a_{j-}^{+}$and $a_{j-}$ are the same operators for the particle with the spin projection $-\frac{1}{2} \hbar ; \hbar$ is Planck's constant, and the particle operator wave functions are normalized by the volume $V$. In the first variant, the interaction Hamiltonian takes the form

$H_{12}=I\left(a_{1+}^{+}+a_{1-}^{+}\right)\left(a_{2+}^{+}+a_{2-}^{+}\right)\left(a_{2+}+a_{2-}\right)\left(a_{1+}+a_{1-}\right)$.

The corresponding interaction constant $I$ equals

$I=\frac{1}{2 V^{2}} \iint d \mathbf{r}_{1} d \mathbf{r}_{2} \Phi\left(\mathbf{r}_{1}-\mathbf{r}_{2}\right)=\frac{3 Q_{1} Q_{2}}{16 \pi \varepsilon_{0} R}$,

where $R$ is the radius of a sphere with the volume $V$. Hereafter, the integrals are calculated by changing to the spherical coordinate frame, and the integration is carried out within a sphere with radius $R$. Then the following expressions for the operators are obtained:

$$
\begin{aligned}
& b_{1+}=b_{1-}=I\left(a_{2+}^{+}+a_{2-}^{+}\right)\left(a_{2+}+a_{2-}\right)\left(a_{1+}+a_{1-}\right), \\
& b_{2+}=b_{2-}=I\left(a_{1+}^{+}+a_{1-}^{+}\right)\left(a_{2+}+a_{2-}\right)\left(a_{1+}+a_{1-}\right), \\
& b_{1+}^{+}=b_{1-}^{+}=I\left(a_{1+}^{+}+a_{1-}^{+}\right)\left(a_{2+}^{+}+a_{2-}^{+}\right)\left(a_{2+}+a_{2-}\right), \\
& b_{2+}^{+}=b_{2-}^{+}=I\left(a_{1+}^{+}+a_{1-}^{+}\right)\left(a_{2+}^{+}+a_{2-}^{+}\right)\left(a_{1+}+a_{1-}\right) .
\end{aligned}
$$

From the triangle form of expansion matrices, it follows that the mean values of all those operators equal zero [1]. From whence, with regard for Eqs. (64) and (66), we obtain the following relations for the mean values of the creation and annihilation operators in the first variant:

$\left\langle a_{1-}\right\rangle=-\left\langle a_{1+}\right\rangle$,

$\left\langle a_{2-}\right\rangle=-\left\langle a_{2+}\right\rangle$,

$\left\langle a_{1-}^{+}\right\rangle=-\left\langle a_{1+}^{+}\right\rangle$,

$\left\langle a_{2-}^{+}\right\rangle=-\left\langle a_{2+}^{+}\right\rangle$.

In the second variant, let us consider the following operator wave functions of the particles:

$\Psi_{1}\left(\mathbf{r}_{1}\right)=\frac{1}{\sqrt{V}}\left(e^{\frac{i \mathbf{p}_{1} \cdot \mathbf{r}_{1}}{\hbar}} a_{1 \mathbf{p}_{1}}+e^{\frac{i \mathbf{q}_{1} \cdot \mathbf{r}_{1}}{\hbar}} a_{1 \mathbf{q}_{1}}\right)$,

$\Psi_{1}^{+}\left(\mathbf{r}_{1}\right)=\frac{1}{\sqrt{V}}\left(e^{\frac{-i \mathbf{p}_{1} \cdot \mathbf{r}_{1}}{\hbar}} a_{1 \mathbf{p}_{1}}^{+}+e^{\frac{-i \mathbf{q}_{1} \cdot \mathbf{r}_{1}}{\hbar}} a_{1 \mathbf{q}_{1}}^{+}\right)$,

$\Psi_{2}\left(\mathbf{r}_{2}\right)=\frac{e^{\frac{i \mathbf{p}_{2} \cdot \mathbf{r}_{2}}{\hbar}}}{\sqrt{V}}\left(a_{2+}+a_{2-}\right)$

$\Psi_{2}^{+}\left(\mathbf{r}_{2}\right)=\frac{e^{\frac{-i \mathbf{p}_{2} \cdot \mathbf{r}_{2}}{\hbar}}}{\sqrt{V}}\left(a_{2+}^{+}+a_{2-}^{+}\right)$

So, in this variant, the first particle has two different states with the different momenta $\mathbf{p}_{1}$ and $\mathbf{q}_{1}$. For the interaction operator, we obtain

$H_{12}=I\left\{a_{1 \mathbf{p}_{1}}^{+}\left(a_{2+}^{+}+a_{2-}^{+}\right)\left(a_{2+}+a_{2-}\right) a_{1 \mathbf{p}_{1}}+\right.$

$\left.+a_{1 \mathbf{q}_{1}}^{+}\left(a_{2+}^{+}+a_{2-}^{+}\right)\left(a_{2+}+a_{2-}\right) a_{1 \mathbf{q}_{1}}\right\}+$

$+I_{1} a_{1 \mathbf{p}_{1}}^{+}\left(a_{2+}^{+}+a_{2-}^{+}\right)\left(a_{2+}+a_{2-}\right) a_{1 \mathbf{q}_{1}}+$

$+I_{2} a_{1 \mathbf{q}_{1}}^{+}\left(a_{2+}^{+}+a_{2-}^{+}\right)\left(a_{2+}+a_{2-}\right) a_{1 \mathbf{p}_{1}}$,

where the interaction constant $I$ is given by formula (93), whereas the interaction constants $I_{1}$ and $I_{2}$ equal

$I_{1}=\frac{1}{2 V^{2}} \iint d \mathbf{r}_{1} d \mathbf{r}_{2} \Phi\left(\mathbf{r}_{1}-\mathbf{r}_{2}\right) e^{\frac{i\left(\mathbf{q}_{1}-\mathbf{p}_{1}\right) \cdot \mathbf{r}_{1}}{\hbar}}=$

$=\frac{2 \pi Q_{1} Q_{2}}{\varepsilon_{0} \Delta_{1}^{5} V^{2}}\left\{1-\cos \left(\Delta_{1} R\right)\right\}\left\{\sin \left(\Delta_{1} R\right)-\right.$

$\left.-\Delta_{1} R \cos \left(\Delta_{1} R\right)\right\}$,

$I_{2}=\frac{1}{2 V^{2}} \iint d \mathbf{r}_{1} d \mathbf{r}_{2} \Phi\left(\mathbf{r}_{1}-\mathbf{r}_{2}\right) e^{\frac{i\left(\mathbf{p}_{1}-\mathbf{q}_{1}\right) \cdot \mathbf{r}_{1}}{\hbar}}=I_{1}$

Here,

$\Delta_{1}=\frac{\left|\mathbf{q}_{1}-\mathbf{p}_{1}\right|}{\hbar}$.

ISSN 2071-0194. Ukr. J. Phys. 2019. Vol. 64, No. 6 
Then we obtain

$$
\begin{aligned}
& b_{1 \mathbf{q}_{1}}=\left(a_{2+}^{+}+a_{2-}^{+}\right)\left(a_{2+}+a_{2-}\right)\left(I a_{1 \mathbf{q}_{1}}+I_{1} a_{1 \mathbf{p}_{1}}\right), \\
& b_{1 \mathbf{p}_{1}}=\left(a_{2+}^{+}+a_{2-}^{+}\right)\left(a_{2+}+a_{2-}\right)\left(I a_{1 \mathbf{p}_{1}}+I_{1} a_{1 \mathbf{q}_{1}}\right), \\
& b_{1 \mathbf{q}_{1}}^{+}=\left(I a_{1 \mathbf{q}_{1}}^{+}+I_{1} a_{1 \mathbf{p}_{1}}^{+}\right)\left(a_{2+}^{+}+a_{2-}^{+}\right)\left(a_{2+}+a_{2-}\right),(112) \\
& b_{1 \mathbf{p}_{1}}^{+}=\left(I a_{1 \mathbf{p}_{1}}^{+}+I_{1} a_{1 \mathbf{q}_{1}}^{+}\right)\left(a_{2+}^{+}+a_{2-}^{+}\right)\left(a_{2+}+a_{2-}\right),(113) \\
& b_{2+}=b_{2-}=\left\{I\left(a_{1 \mathbf{p}_{1}}^{+} a_{1 \mathbf{p}_{1}}+a_{1 \mathbf{q}_{1}}^{+} a_{1 \mathbf{q}_{1}}\right)+\right. \\
& \left.+I_{1}\left(a_{1 \mathbf{p}_{1}}^{+} a_{1 \mathbf{q}_{1}}+a_{1 \mathbf{q}_{1}}^{+} a_{1 \mathbf{p}_{1}}\right)\right\}\left(a_{2+}+a_{2-}\right), \\
& b_{2+}^{+}=b_{2-}^{+}=\left(a_{2+}^{+}+a_{2-}^{+}\right)\left\{I\left(a_{1 \mathbf{p}_{1}}^{+} a_{1 \mathbf{p}_{1}}+a_{1 \mathbf{q}_{1}}^{+} a_{1 \mathbf{q}_{1}}\right)+\right. \\
& \left.+I_{1}\left(a_{1 \mathbf{p}_{1}}^{+} a_{1 \mathbf{q}_{1}}+a_{1 \mathbf{q}_{1}}^{+} a_{1 \mathbf{p}_{1}}\right)\right\} .
\end{aligned}
$$

From Eqs. (114) and (115) in view of Eqs. (64) and (66), we have

$$
\begin{aligned}
& \left\langle a_{2-}\right\rangle=-\left\langle a_{2+}\right\rangle, \\
& \left\langle a_{2-}^{+}\right\rangle=-\left\langle a_{2+}^{+}\right\rangle .
\end{aligned}
$$

On the other hand, from Eqs. (110) and (111) in view of Eq. (64), we obtain

$I\left\langle a_{1 \mathbf{q}_{1}}\right\rangle+I_{1}\left\langle a_{1 \mathbf{p}_{1}}\right\rangle=0$,
$I\left\langle a_{1 \mathbf{p}_{1}}\right\rangle+I_{1}\left\langle a_{1 \mathbf{q}_{1}}\right\rangle=0$.

From whence, it follows that

$\left\{1-\cos \left(\Delta_{1} R\right)\right\}\left\{\Delta_{1} R \cos \left(\Delta_{1} R\right)-\sin \left(\Delta_{1} R\right)\right\}=\frac{\left(\Delta_{1} R\right)^{5}}{6}$.

The solution of this equation reads

$\Delta_{1} R=C=1.3680427635$,

which corresponds to the condition

$I_{1}=-I$,

so that

$\left\langle a_{1 \mathbf{q}_{1}}\right\rangle=\left\langle a_{1 \mathbf{p}_{1}}\right\rangle$.

The following relation can be derived analogously:

$\left\langle a_{1 \mathbf{q}_{1}}^{+}\right\rangle=\left\langle a_{1 \mathbf{p}_{1}}^{+}\right\rangle$.

The third variant can be obtained from the second one by permutating the subscripts indicating the particle kinds and substituting $\Delta_{1}$ by

$\Delta_{2}=\frac{\left|\mathbf{q}_{2}-\mathbf{p}_{2}\right|}{\hbar}$.

ISSN 2071-0194. Ukr. J. Phys. 2019. Vol. 64, No. 6
For the fourth variant, the operator wave functions are taken in the form

$$
\begin{aligned}
& \Psi_{1}\left(\mathbf{r}_{1}\right)=\frac{1}{\sqrt{V}}\left(e^{\frac{i \mathbf{p}_{1} \cdot \mathbf{r}_{1}}{\hbar}} a_{1 \mathbf{p}_{1}}+e^{\frac{i \mathbf{q}_{1} \cdot \mathbf{r}_{1}}{\hbar}} a_{1 \mathbf{q}_{1}}\right), \\
& \Psi_{1}^{+}\left(\mathbf{r}_{1}\right)=\frac{1}{\sqrt{V}}\left(e^{\frac{-i \mathbf{p}_{1} \cdot \mathbf{r}_{1}}{\hbar}} a_{1 \mathbf{p}_{1}}^{+}+e^{\frac{-i \mathbf{q}_{1} \cdot \mathbf{r}_{1}}{\hbar}} a_{1 \mathbf{q}_{1}}^{+}\right), \\
& \Psi_{2}\left(\mathbf{r}_{2}\right)=\frac{1}{\sqrt{V}}\left(e^{\frac{i \mathbf{p}_{2} \cdot \mathbf{r}_{2}}{\hbar}} a_{2 \mathbf{p}_{2}}+e^{\frac{i \mathbf{q}_{2} \cdot \mathbf{r}_{2}}{\hbar}} a_{2 \mathbf{q}_{2}}\right), \\
& \Psi_{2}^{+}\left(\mathbf{r}_{2}\right)=\frac{1}{\sqrt{V}}\left(e^{\frac{-i \mathbf{p}_{2} \cdot \mathbf{r}_{2}}{\hbar}} a_{2 \mathbf{p}_{2}}^{+}+e^{\frac{-i \mathbf{q}_{2} \cdot \mathbf{r}_{2}}{\hbar}} a_{2 \mathbf{q}_{2}}^{+}\right) .
\end{aligned}
$$

Then, for the interaction operator, we obtain

$H_{12}=I\left(a_{1 \mathbf{p}_{1}}^{+} a_{2 \mathbf{p}_{2}}^{+} a_{2 \mathbf{p}_{2}} a_{1 \mathbf{p}_{1}}+a_{1 \mathbf{q}_{1}}^{+} a_{2 \mathbf{q}_{2}}^{+} a_{2 \mathbf{q}_{2}} a_{1 \mathbf{q}_{1}}+\right.$

$\left.+a_{1 \mathbf{p}_{1}}^{+} a_{2 \mathbf{q}_{2}}^{+} a_{2 \mathbf{q}_{2}} a_{1 \mathbf{p}_{1}}+a_{1 \mathbf{q}_{1}}^{+} a_{2 \mathbf{p}_{2}}^{+} a_{2 \mathbf{p}_{2}} a_{1 \mathbf{q}_{1}}\right)+$

$+I_{1}\left(a_{1 \mathbf{p}_{1}}^{+} a_{2 \mathbf{p}_{2}}^{+} a_{2 \mathbf{p}_{2}} a_{1 \mathbf{q}_{1}}+a_{1 \mathbf{p}_{1}}^{+} a_{2 \mathbf{q}_{2}}^{+} a_{2 \mathbf{q}_{2}} a_{1 \mathbf{q}_{1}}\right)+$

$+I_{2}\left(a_{1 \mathbf{q}_{1}}^{+} a_{2 \mathbf{p}_{2}}^{+} a_{2 \mathbf{p}_{2}} a_{1 \mathbf{p}_{1}}+a_{1 \mathbf{q}_{1}}^{+} a_{2 \mathbf{q}_{2}}^{+} a_{2 \mathbf{q}_{2}} a_{1 \mathbf{p}_{1}}\right)+$

$+I_{3}\left(a_{1 \mathbf{p}_{1}}^{+} a_{2 \mathbf{p}_{2}}^{+} a_{2 \mathbf{q}_{2}} a_{1 \mathbf{p}_{1}}+a_{1 \mathbf{q}_{1}}^{+} a_{2 \mathbf{p}_{2}}^{+} a_{2 \mathbf{q}_{2}} a_{1 \mathbf{q}_{1}}\right)+$

$+I_{4}\left(a_{1 \mathbf{p}_{1}}^{+} a_{2 \mathbf{q}_{2}}^{+} a_{2 \mathbf{p}_{2}} a_{1 \mathbf{p}_{1}}+a_{1 \mathbf{q}_{1}}^{+} a_{2 \mathbf{q}_{2}}^{+} a_{2 \mathbf{p}_{2}} a_{1 \mathbf{q}_{1}}\right)+$

$+I_{5} a_{1 \mathbf{p}_{1}}^{+} a_{2 \mathbf{q}_{2}}^{+} a_{2 \mathbf{p}_{2}} a_{1 \mathbf{q}_{1}}+I_{6} a_{1 \mathbf{q}_{1}}^{+} a_{2 \mathbf{p}_{2}}^{+} a_{2 \mathbf{q}_{2}} a_{1 \mathbf{p}_{1}}+$

$+I_{7} a_{1 \mathbf{q}_{1}}^{+} a_{2 \mathbf{q}_{2}}^{+} a_{2 \mathbf{p}_{2}} a_{1 \mathbf{p}_{1}}+I_{8} a_{1 \mathbf{p}_{1}}^{+} a_{2 \mathbf{p}_{2}}^{+} a_{2 \mathbf{q}_{2}} a_{1 \mathbf{q}_{1}}$.

Here, the interaction constants $I, I_{1}$, and $I_{2}$ are given by expressions (93), (107), and (108), respectively, whereas the other interaction constants equal

$I_{3}=\frac{1}{2 V^{2}} \iint d \mathbf{r}_{1} d \mathbf{r}_{2} \Phi\left(\mathbf{r}_{1}-\mathbf{r}_{2}\right) e^{\frac{i\left(\mathbf{q}_{2}-\mathbf{p}_{2}\right) \cdot \mathbf{r}_{2}}{\hbar}}=$

$=\frac{2 \pi Q_{1} Q_{2}}{\varepsilon_{0} \Delta_{2}^{5} V^{2}}\left\{1-\cos \left(\Delta_{2} R\right)\right\}\left\{\sin \left(\Delta_{2} R\right)-\right.$

$\left.-\Delta_{2} R \cos \left(\Delta_{2} R\right)\right\}$,

$I_{4}=\frac{1}{2 V^{2}} \iint d \mathbf{r}_{1} d \mathbf{r}_{2} \Phi\left(\mathbf{r}_{1}-\mathbf{r}_{2}\right) e^{\frac{i\left(\mathbf{p}_{2}-\mathbf{q}_{2}\right) \cdot \mathbf{r}_{2}}{\hbar}}=I_{3}$,

$I_{5}=\frac{1}{2 V^{2}} \iint d \mathbf{r}_{1} d \mathbf{r}_{2} \Phi\left(\mathbf{r}_{1}-\mathbf{r}_{2}\right) e^{i \frac{\left(\mathbf{q}_{1}-\mathbf{p}_{1}\right) \cdot \mathbf{r}_{1}+\left(\mathbf{p}_{2}-\mathbf{q}_{2}\right) \cdot \mathbf{r}_{2}}{\hbar}}=$

$=\frac{2 \pi Q_{1} Q_{2}}{\varepsilon_{0} \Delta_{1}^{2}\left|\boldsymbol{\Delta}_{1}-\boldsymbol{\Delta}_{2}\right|^{3} V^{2}}\left\{1-\cos \left(\Delta_{1} R\right)\right\} \times$

$\times\left\{\sin \left(\left|\boldsymbol{\Delta}_{1}-\boldsymbol{\Delta}_{2}\right| R\right)-\left|\boldsymbol{\Delta}_{1}-\boldsymbol{\Delta}_{2}\right| \times\right.$

$\left.\times R \cos \left(\left|\boldsymbol{\Delta}_{1}-\boldsymbol{\Delta}_{2}\right| R\right)\right\}$. 
Here, we denoted

$\boldsymbol{\Delta}_{i}=\frac{\mathbf{q}_{i}-\mathbf{p}_{i}}{\hbar}$.

$I_{6}=\frac{1}{2 V^{2}} \iint d \mathbf{r}_{1} d \mathbf{r}_{2} \Phi\left(\mathbf{r}_{1}-\mathbf{r}_{2}\right) \times$

$\times e^{i \frac{\left(\mathbf{p}_{1}-\mathbf{q}_{1}\right) \cdot \mathbf{r}_{1}+\left(\mathbf{q}_{2}-\mathbf{p}_{2}\right) \cdot \mathbf{r}_{2}}{\hbar}}=I_{5}$,

$I_{7}=\frac{1}{2 V^{2}} \iint d \mathbf{r}_{1} d \mathbf{r}_{2} \Phi\left(\mathbf{r}_{1}-\mathbf{r}_{2}\right) e^{i \frac{\left(\mathbf{p}_{1}-\mathbf{q}_{1}\right) \cdot \mathbf{r}_{1}+\left(\mathbf{p}_{2}-\mathbf{q}_{2}\right) \cdot \mathbf{r}_{2}}{\hbar}}=$

$=\frac{2 \pi Q_{1} Q_{2}}{\varepsilon_{0} \Delta_{1}^{2}\left|\boldsymbol{\Delta}_{1}+\boldsymbol{\Delta}_{2}\right|^{3} V^{2}}\left\{1-\cos \left(\Delta_{1} R\right)\right\} \times$

$\times\left\{\sin \left(\left|\boldsymbol{\Delta}_{1}+\boldsymbol{\Delta}_{2}\right| R\right)-\left|\boldsymbol{\Delta}_{1}+\boldsymbol{\Delta}_{2}\right| \times\right.$

$\left.\times R \cos \left(\left|\boldsymbol{\Delta}_{1}+\boldsymbol{\Delta}_{2}\right| R\right)\right\}$,

$I_{8}=\frac{1}{2 V^{2}} \iint d \mathbf{r}_{1} d \mathbf{r}_{2} \Phi\left(\mathbf{r}_{1}-\mathbf{r}_{2}\right) \times$

$\times e^{i \frac{\left(\mathbf{q}_{1}-\mathbf{p}_{1}\right) \cdot \mathbf{r}_{1}+\left(\mathbf{q}_{2}-\mathbf{p}_{2}\right) \cdot \mathbf{r}_{2}}{\hbar}}=I_{7}$.

From the Hamiltonian, we find

$$
\begin{aligned}
& b_{1 \mathbf{q}_{1}}=\left(a_{2 \mathbf{p}_{2}}^{+} a_{2 \mathbf{p}_{2}}+a_{2 \mathbf{q}_{2}}^{+} a_{2 \mathbf{q}_{2}}\right)\left(I a_{1 \mathbf{q}_{1}}+I_{2} a_{1 \mathbf{p}_{1}}\right)+ \\
& +a_{2 \mathbf{p}_{2}}^{+} a_{2 \mathbf{q}_{2}}\left(I_{3} a_{1 \mathbf{q}_{1}}+I_{6} a_{1 \mathbf{p}_{1}}\right)+ \\
& +a_{2 \mathbf{q}_{2}}^{+} a_{2 \mathbf{p}_{2}}\left(I_{4} a_{1 \mathbf{q}_{1}}+I_{7} a_{1 \mathbf{p}_{1}}\right) \text {, } \\
& b_{1 \mathbf{p}_{1}}=\left(a_{2 \mathbf{p}_{2}}^{+} a_{2 \mathbf{p}_{2}}+a_{2 \mathbf{q}_{2}}^{+} a_{2 \mathbf{q}_{2}}\right)\left(I a_{1 \mathbf{p}_{1}}+I_{1} a_{1 \mathbf{q}_{1}}\right)+ \\
& +a_{2 \mathbf{p}_{2}}^{+} a_{2 \mathbf{q}_{2}}\left(I_{3} a_{1 \mathbf{p}_{1}}+I_{8} a_{1 \mathbf{q}_{1}}\right)+ \\
& +a_{2 \mathbf{q}_{2}}^{+} a_{2 \mathbf{p}_{2}}\left(I_{4} a_{1 \mathbf{p}_{1}}+I_{5} a_{1 \mathbf{q}_{1}}\right) \text {, } \\
& b_{2 \mathbf{q}_{2}}=\left(a_{1 \mathbf{p}_{1}}^{+} a_{1 \mathbf{p}_{1}}+a_{1 \mathbf{q}_{1}}^{+} a_{1 \mathbf{q}_{1}}\right)\left(I a_{2 \mathbf{q}_{2}}+I_{4} a_{2 \mathbf{p}_{2}}\right)+ \\
& +a_{1 \mathbf{p}_{1}}^{+} a_{1 \mathbf{q}_{1}}\left(I_{1} a_{2 \mathbf{q}_{2}}+I_{5} a_{2 \mathbf{p}_{2}}\right)+ \\
& +a_{1 \mathbf{q}_{1}}^{+} a_{1 \mathbf{p}_{1}}\left(I_{2} a_{2 \mathbf{q}_{2}}+I_{7} a_{2 \mathbf{p}_{2}}\right) \\
& b_{2 \mathbf{p}_{2}}=\left(a_{1 \mathbf{p}_{1}}^{+} a_{1 \mathbf{p}_{1}}+a_{1 \mathbf{q}_{1}}^{+} a_{1 \mathbf{q}_{1}}\right)\left(I a_{2 \mathbf{p}_{2}}+I_{3} a_{2 \mathbf{q}_{2}}\right)+ \\
& +a_{1 \mathbf{p}_{1}}^{+} a_{1 \mathbf{q}_{1}}\left(I_{1} a_{2 \mathbf{p}_{2}}+I_{8} a_{2 \mathbf{q}_{2}}\right)+ \\
& +a_{1 \mathbf{q}_{1}}^{+} a_{1 \mathbf{p}_{1}}\left(I_{2} a_{2 \mathbf{p}_{2}}+I_{6} a_{2 \mathbf{q}_{2}}\right) \text {, } \\
& b_{1 \mathbf{q}_{1}}^{+}=\left(I a_{1 \mathbf{q}_{1}}^{+}+I_{1} a_{1 \mathbf{p}_{1}}^{+}\right)\left(a_{2 \mathbf{p}_{2}}^{+} a_{2 \mathbf{p}_{2}}+a_{2 \mathbf{q}_{2}}^{+} a_{2 \mathbf{q}_{2}}\right)+ \\
& +\left(I_{3} a_{1 \mathbf{q}_{1}}^{+}+I_{8} a_{1 \mathbf{p}_{1}}^{+}\right) a_{2 \mathbf{p}_{2}}^{+} a_{2 \mathbf{q}_{2}}+ \\
& +\left(I_{4} a_{1 \mathbf{q}_{1}}^{+}+I_{5} a_{1 \mathbf{p}_{1}}^{+}\right) a_{2 \mathbf{q}_{2}}^{+} a_{2 \mathbf{p}_{2}} \text {, } \\
& b_{1 \mathbf{p}_{1}}^{+}=\left(I a_{1 \mathbf{p}_{1}}^{+}+I_{2} a_{1 \mathbf{q}_{1}}^{+}\right)\left(a_{2 \mathbf{p}_{2}}^{+} a_{2 \mathbf{p}_{2}}+a_{2 \mathbf{q}_{2}}^{+} a_{2 \mathbf{q}_{2}}\right)+ \\
& +\left(I_{3} a_{1 \mathbf{p}_{1}}^{+}+I_{6} a_{1 \mathbf{q}_{1}}^{+}\right) a_{2 \mathbf{p}_{2}}^{+} a_{2 \mathbf{q}_{2}}+ \\
& +\left(I_{4} a_{1 \mathbf{p}_{1}}^{+}+I_{7} a_{1 \mathbf{q}_{1}}^{+}\right) a_{2 \mathbf{q}_{2}}^{+} a_{2 \mathbf{p}_{2}} \text {, } \\
& b_{2 \mathbf{q}_{2}}^{+}=\left(I a_{2 \mathbf{q}_{2}}^{+}+I_{3} a_{2 \mathbf{p}_{2}}^{+}\right)\left(a_{1 \mathbf{p}_{1}}^{+} a_{1 \mathbf{p}_{1}}+a_{1 \mathbf{q}_{1}}^{+} a_{1 \mathbf{q}_{1}}\right)+ \\
& +\left(I_{1} a_{2 \mathbf{q}_{2}}^{+}+I_{8} a_{2 \mathbf{p}_{2}}^{+}\right) a_{1 \mathbf{p}_{1}}^{+} a_{1 \mathbf{q}_{1}}+ \\
& +\left(I_{2} a_{2 \mathbf{q}_{2}}^{+}+I_{6} a_{2 \mathbf{p}_{2}}^{+}\right) a_{1 \mathbf{q}_{1}}^{+} a_{1 \mathbf{p}_{1}} \text {, }
\end{aligned}
$$

$$
\begin{aligned}
& b_{2 \mathbf{p}_{2}}^{+}=\left(I a_{2 \mathbf{p}_{2}}^{+}+I_{4} a_{2 \mathbf{q}_{2}}^{+}\right)\left(a_{1 \mathbf{p}_{1}}^{+} a_{1 \mathbf{p}_{1}}+a_{1 \mathbf{q}_{1}}^{+} a_{1 \mathbf{q}_{1}}\right)+ \\
& +\left(I_{1} a_{2 \mathbf{p}_{2}}^{+}+I_{5} a_{2 \mathbf{q}_{2}}^{+}\right) a_{1 \mathbf{p}_{1}}^{+} a_{1 \mathbf{q}_{1}}+ \\
& +\left(I_{2} a_{2 \mathbf{p}_{2}}^{+}+I_{7} a_{2 \mathbf{q}_{2}}^{+}\right) a_{1 \mathbf{q}_{1}}^{+} a_{1 \mathbf{p}_{1}} .
\end{aligned}
$$

As was done above, we obtain

$$
\left\{1-\cos \left(\Delta_{i} R\right)\right\}\left\{\Delta_{i} R \cos \left(\Delta_{i} R\right)-\sin \left(\Delta_{i} R\right)\right\}=\frac{\left(\Delta_{i} R\right)^{5}}{6},
$$

$\Delta_{2} R=\Delta_{1} R=C=1.3680427635$.

One can see that $R$ is a definite characteristic radius, whose magnitude is reciprocal to the difference between the vectors of two possible momenta for the particles. This difference turns out identical in magnitude for both particles. Finally, we obtain

$$
\begin{aligned}
& I_{3}=I_{1}=-I, \\
& \left\langle a_{i \mathbf{q}_{i}}\right\rangle=\left\langle a_{i \mathbf{p}_{i}}\right\rangle, \\
& \left\langle a_{i \mathbf{q}_{i}}^{+}\right\rangle=\left\langle a_{i \mathbf{p}_{i}}^{+}\right\rangle .
\end{aligned}
$$

\section{Conclusions}

As one can see, the DAM is effective for finding the correlation functions of the third order. Using results (64) and (66) obtained in the third order, we also found relations for the mean values of the creation and annihilation operators for various forms of the interaction operator. Hence, there is a specific hybridization of quantities belonging to higher and lower orders, which makes it possible to determine quantities that remained uncertain earlier.

On the other hand, we saw how the zeroing of the mean values of the expansion-basis operators $b$ obtained in the first order brought about a result for the correlation functions in the third order. At the same time, the relations obtained for the correlation functions in the third order and including the same expansion-basis operators will give rise to the corresponding effect in the fifth order of correlation functions. Thus, there are all grounds to hope for that all lower-order correlation functions, which are required to calculate the mean values of physical quantities for a Coulomb pair, can be determined in turn.

Furthermore, the values obtained for the normal particle distribution functions in the second order made it possible to determine the main forms of the particle interaction operator, which depend on the combination of possible values for the momenta

ISSN 2071-0194. Ukr. J. Phys. 2019. Vol. 64, No. 6 
and spin projections of the particles. Note that, in the last variant, as well as in the previous two, we did not consider the issue concerning the projections of particle spins in various possible states with different particle momenta. Those projections can be both identical and different! Only the states with identical momenta demand that the spin projections should be different in order that those states could be considered as different states of the corresponding particles. This strange conclusion follows from the fact that the Coulomb interaction does not depend on the spin. Therefore, this issue has to be studied further.

Thus, there emerges a clear scheme of calculations to an arbitrary order. First, we find the equations of motion for the creation and annihilation operators. Those equations, with the help of expressions for the commutators or anticommutators, are used to obtain equations for the operators themselves in a definite order. Then, by applying Eq. (40), the relations for the correlation functions and the central distribution moments that are independent of the interaction operator form are found. These relations are appended with the relations that follow from the operator equations that were obtained earlier. In addition, from the lower-order relations obtained for the correlation functions and including the basis expansion operators $b$, new relations are found for the corresponding correlation functions, which directly include the operators of particle creation and annihilation. Finally, the corresponding relations that follow from explicit expressions for the specific interaction operator forms are considered.

The growth in the order of correlation functions does not change this general calculation procedure. Technically, the calculations become more complicated owing to an increase in the number of correlations which are to be considered and the number of equations which are to be solved. However, every order has its own specificity associated with the physical meaning of the corresponding correlation functions. For instance, the average value of the interaction energy is determined by the correlation functions of the fourth order.

\section{APPENDIX}

Let us consider how the equations of motion for the creation and annihilation operators can be used to derive the equations for the third-order operators themselves. For example, taking into account that

$\left[a_{i p}^{+}, a_{i q}\right]_{+} a_{j}=a_{j} \delta_{p q}$

some important relations can be obtained. In particular, from Eq. (12), we find

$\left[a_{i p}^{+}, b_{i q}\right]_{+} a_{j}=\left[b_{i p}^{+}, a_{i q}\right]_{+} a_{j}$.

Now, calculating the commutator of Eq. (152) with the Hamiltonian, we obtain

$2\left[b_{i p}^{+}, b_{i q}\right]_{+} a_{j}=\left(\left[a_{i p}^{+}, b_{i q}\right]_{+}-\left[b_{i p}^{+}, a_{i q}\right]_{+}\right) b_{j}+$

$+\left(K_{22 p}^{(i)}+K_{22 q}^{(i)}-\varepsilon_{i p}-\varepsilon_{i q}\right)\left[a_{i p}^{+}, b_{i q}\right]_{+} a_{j}$.

In the same way, we obtain the equations

$\left[a_{i p}^{+}, a_{i q}\right]_{+} a_{j}^{+}=a_{j}^{+} \delta_{p q}$,

$\left[a_{i p}^{+}, b_{i q}\right]_{+} a_{j}^{+}=\left[b_{i p}^{+}, a_{i q}\right]_{+} a_{j}^{+}$

$2\left[b_{i p}^{+}, b_{i q}\right]_{+} a_{j}^{+}=\left(\left[b_{i p}^{+}, a_{i q}\right]_{+}-\left[a_{i p}^{+}, b_{i q}\right]_{+}\right) b_{j}^{+}+$

$+\left(K_{22 p}^{(i)}+K_{22 q}^{(i)}-\varepsilon_{i p}-\varepsilon_{i q}\right)\left[a_{i p}^{+}, b_{i q}\right]_{+} a_{j}^{+}$,

$a_{j}\left[a_{i p}^{+}, a_{i q}\right]_{+}=a_{j} \delta_{p q}$,

$a_{j}\left[a_{i p}^{+}, b_{i q}\right]_{+}=a_{j}\left[b_{i p}^{+}, a_{i q}\right]_{+}$,

$2 a_{j}\left[b_{i p}^{+}, b_{i q}\right]_{+}=b_{j}\left(\left[a_{i p}^{+}, b_{i q}\right]_{+}-\left[b_{i p}^{+}, a_{i q}\right]_{+}\right)+$

$+\left(K_{22 p}^{(i)}+K_{22 q}^{(i)}-\varepsilon_{i p}-\varepsilon_{i q}\right) a_{j}\left[a_{i p}^{+}, b_{i q}\right]_{+}$,

$a_{j}^{+}\left[a_{i p}^{+}, a_{i q}\right]_{+}=a_{j}^{+} \delta_{p q}$,

$a_{j}^{+}\left[a_{i p}^{+}, b_{i q}\right]_{+}=a_{j}^{+}\left[b_{i p}^{+}, a_{i q}\right]_{+}$,

$2 a_{j}^{+}\left[b_{i p}^{+}, b_{i q}\right]_{+}=b_{j}^{+}\left(\left[b_{i p}^{+}, a_{i q}\right]_{+}-\left[a_{i p}^{+}, b_{i q}\right]_{+}\right)+$

$+\left(K_{22 p}^{(i)}+K_{22 q}^{(i)}-\varepsilon_{i p}-\varepsilon_{i q}\right) a_{j}^{+}\left[a_{i p}^{+}, b_{i q}\right]_{+}$,

$a_{i p}^{+}\left[a_{i q}, a_{j}\right]=0$,

$a_{i p}^{+}\left[b_{i q}, a_{j}\right]=-a_{i p}^{+}\left[a_{i q}, b_{j}\right]$,

$2 a_{i p}^{+}\left[b_{i q}, b_{j}\right]=b_{i p}^{+}\left(\left[a_{i q}, b_{j}\right]+\left[b_{i q}, a_{j}\right]\right)+$

$+\left(\varepsilon_{j}-\varepsilon_{i q}+K_{22 q}^{(i)}-K_{22}^{j}\right) a_{i p}^{+}\left[a_{i q}, b_{j}\right]$,

$a_{i p}^{+}\left[a_{i q}, a_{j}^{+}\right]=0$,

$a_{i p}^{+}\left[b_{i q}, a_{j}^{+}\right]=a_{i p}^{+}\left[a_{i q}, b_{j}^{+}\right]$,

$2 a_{i p}^{+}\left[b_{i q}, b_{j}^{+}\right]=b_{i p}^{+}\left(\left[a_{i q}, b_{j}^{+}\right]-\left[b_{i q}, a_{j}^{+}\right]\right)+$

$+\left(K_{22 q}^{(i)}+K_{22}^{(j)}-\varepsilon_{i q}-\varepsilon_{j}\right) a_{i p}^{+}\left[a_{i q}, b_{j}^{+}\right]$,

$\left[a_{i p}^{+}, a_{j}\right] a_{i q}=0$,

$\left[b_{i p}^{+}, a_{j}\right] a_{i q}=\left[a_{i p}^{+}, b_{j}\right] a_{i q}$,

$2\left[b_{i p}^{+}, b_{j}\right] a_{i q}=\left(\left[a_{i p}^{+}, b_{j}\right]-\left[b_{i p}^{+}, a_{j}\right]\right) b_{i q}+$

$+\left(K_{22 p}^{(i)}-\varepsilon_{i p}+K_{22}^{(j)}-\varepsilon_{j}\right)\left[a_{i p}^{+}, b_{j}\right] a_{i q}$, 
$\left[a_{i q}, a_{j}^{+}\right] a_{i p}^{+}=0$,

$\left[b_{i q}, a_{j}^{+}\right] a_{i p}^{+}=\left[a_{i q}, b_{j}^{+}\right] a_{i p}^{+}$,

$2\left[b_{i q}, b_{j}^{+}\right] a_{i p}^{+}=\left(\left[a_{i q}, b_{j}^{+}\right]-\left[b_{i q}, a_{j}^{+}\right]\right) b_{i p}^{+}+$

$+\left(K_{22 q}^{(i)}+K_{22}^{(j)}-\varepsilon_{i q}-\varepsilon_{j}\right)\left[b_{i q}, a_{j}^{+}\right] a_{i p}^{+}$,

and so on.

1. V.I. Vaskivskyi. Correlation functions of Coulomb pair. Ukr. Fiz. Zh. 60, 1156 (2015) (in Ukrainian).

2. M.F. Sarry. Analytical methods for calculating correlation functions in quantum statistical physics. Usp. Fiz. Nauk 161, 47 (1991) (in Russian).

3. A.I. Akhiezer, V.V. Krasilnikov et al. Theory of superfluid Fermi-liquid. Usp. Fiz. Nauk 163, No. 2, 1 (1993) (in Russian).

4. A.I. Akhiezer, V.V. Krasil'nikov, S.V. Peletminskii, A.A. Yatsenko. Research on superfluidity and superconductivity on the basis of the Fermi liquid concept. Phys. Rep. 245, 1 (1994).

5. A.I. Akhiezer, A.A. Isaev, S.V. Peletminskii, A.P. Rekalo, A.A. Yatsenko. On the theory of superfluidity of nuclear matter on the basis of the Fermi-liquid approach. Zh. Ekksp. Teor. Fiz. 112, 3 (1997) (in Russian).

6. V.R. Shaginyan, M.Ya. Amus'ya, K.G. Popov. Universal behavior of strongly correlated Fermi systems. Usp. Fiz. Nauk 177, 585 (2007) (in Russian).

7. V.I. Belyavsky, Yu.V. Kopaev. Superconductivity of repulsing particles. Usp. Fiz. Nauk 176, 457 (2006) (in Russian).
8. V.O. Krasnov. Fermion spectrum of Bose-Fermi-Hubbard model in the phase with Bose-Einstein condensate. Ukr. J. Phys. 60, 443 (2015).

9. I. Bariakhtar, A. Nazarenko. A model for $d_{x_{2}-y_{2}}$ superconductivity in the strongly correlated fermionic system. Ukr. J. Phys. 59, 487 (2014).

10. N.N. Bogolubov, N.N. Bogolubov, jr., Introduction to Quantum Statistical Mechanics (Gordon and Breach, 1992).

11. A.Z. Patashinski, V.L. Pokrovski. Fluctuation Theory of Phase Transitions (Pergamon Press, 1982).

Received 18.02.15

Translated from Ukrainian by O.I. Voitenko

\section{В.I. Васъківсъкий}

\section{КОРЕЛЯЩЙНІ ФУНКЦІї}

КУЛОНІВСЬКОЇ ПАРИ ТРЕТЬОГО ПОРЯДКУ

$\mathrm{P}$ е $з$ ю м е

В статті вперше публікуються результати для кореляційних функцій третього порядку для випадку двох частинок, що підлягають електростатичній взаємодії, отримані прямим алгебраїчним методом знаходження кореляційних функцій. Розглянуті як основні співвідношення для цих кореляційних функцій, що не залежать від явного вигляду потенціалу взаємодії частинок, так і 4 види форм оператора взаємодії та співвідношення для кореляційних функцій, які для них виникають. 